\title{
Design of a Methane Concentration Detector Based on Spectrum Absorption
}

\author{
Wenjuan Cheng \\ School of Computer and Information, \\ Hefei University of Technology, \\ Hefei, China \\ e-mail: cheng@ah.edu.cn
}

\author{
Junguang Han \\ School of Computer and Information, \\ Hefei University of Technology, \\ Hefei, China \\ e-mail: 1220498135@qq.com
}

\author{
Yongzhong $\mathrm{Wu}$ \\ School of Computer and Information, \\ Hefei University of Technology, \\ Hefei, China \\ e-mail: wyz64@163.com
}

\begin{abstract}
It is necessary to have a methane concentration detector in the coal mine workspace. In this paper, a methane detector is designed which is based on non-dispersive infrared spectrum absorption theory and employs the single-light beam and double wavelength differential detection technology and integrative infrared filter detector. Performance test for this system has been conducted, and the experimental data has been fitted and relation formula between methane concentration and voltage under different temperature has been given .The results of experiment indicates that the detector can effectively detected methane concentration and had lower measurement error and higher detection sensitivity and can be primarily used in coal mine.
\end{abstract}

Keywords-optical path; pyroelectric detector; NDIR; temperature measurement; concentration detection

\section{INTRODUCTION}

With the intensification of energy demand all over the world, lots of coal-producing area's scale is expanding and the number of high concentration gas mine thus increases greatly. As a result, the mine disaster resulting from methane explosion occurs constantly. Therefore, people have developed different kinds of methane concentration detector, such as catalyzing burning methane detector, semiconductor gas-sensitive detector, optical interference methane detector and advanced laser methane detector. They can detect the methane concentration on some level, but they also have different kinds of shortcomings. Catalyzing burning methane detector is easily poisoning and needs continually adjustment; semiconductor gas-sensitive detector has low detection precision; optical interference methane detector is easily interfered by non-target gas and other environmental factors; laser methane detector is expensive and so on.

In order to avoid the shortcomings described above, a methane detector which is based on nondispersive infrared (NDIR) spectrum absorption theory and uses the single-light beam and double wavelength differential detection technology is designed in this paper.

\section{DETECTION PRINCIPLE OF INFraRED METHANE DETECTOR}

Infrared methane detector design is based on the theory that different gas has different infrared absorption spectrum and the same gas of different concentration has different extent of absorption to infrared light as shown in Figure 1(a) and 1(b).

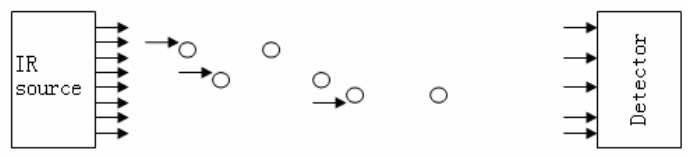

Figure 1(a)

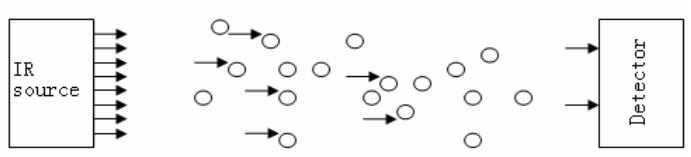

Figure 1(b)

Concentration value of absorbed gas follows the Lamber-Beer law:

$$
\mathrm{I}=\mathrm{I}_{0} \mathrm{e}^{-\mu \mathrm{cl}}
$$

Where, $\mathrm{I}$ is the intensity of transmission light. $\mathrm{I}_{0}$ is the intensity of incident light. $l$ is the length of the absorption path. $\mathrm{c}$ is concentration of methane. $\mu$ is absorption coefficient. Different gas has different absorption coefficient, while for the same gas, $\mu$ signifies wavelength's function. Therefore, we can identify whether the target gas exists or not and obtain its concentration through measuring the absorbed light intensity.

Each kind of gas has the specific absorption spectrum and absorbs little from other wavelength's light. In order to eliminate the effect of non-target gases and the instability of light radiation on the measurement, the design of the methane detector employs the single-light beam and double wavelength differential detection technology and introduces reference light which can't be absorbed by target detector.

It can be concluded from the HITRAN database that methane gas at a wavelength of $3.31 \mu \mathrm{m}$ absorption ability of light is the strongest, but there is almost no absorption at 
$4.00 \mu \mathrm{m}$, light of $4.00 \mu \mathrm{m}$ wavelength is thus selected as reference light.

\section{SYSTEM HARDWARE DESIGN}

System hardware design includes two parts: optical path design and circuit design.

\section{A. Infrared Methane Concentration Detector Optical Path Design}

The NDIR set-up applies one light source, two detectors and a barrel-type gas cell. Figure 2 shows the schematic arrangements.

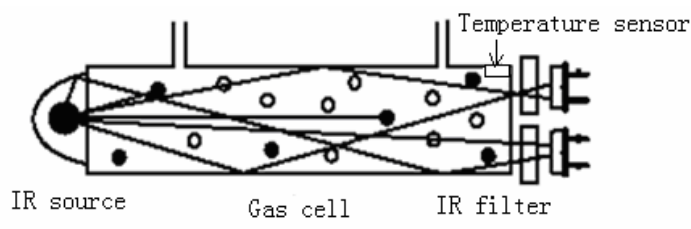

Figure 2. Single light source and dual detectors of model

As for mixed gases, in order to analyse the specific components, a suitable analysis of gas absorption wavelength narrowband filter should be installed in front of pyroelectric detector, making pyroelectric detector only reflect the change of target gas's concentration. Firstly, the infrared light is absorbed by the target gas, then the light passes the specific filter, at last the light enters into the pyroelectric detector and the signal strength of detector reflects the absorbing ability of the target gas.

But the light's propagation path is so complicated that part some is scattered, some is shot at right angles and other is diffuse reflection. If traditional Lamber-Beer law is applied as the basis of quantitative analysis, only direct light can be utilized and other light's contribution to pyroelectric detector is neglected. Part of diffuse light could irradiate to pyroelectric detector, it will cause error if the formula (1) is used directly. In order to use all light that can reach the filter and reduce error, this paper uses the method mentioned in document [10]:

$$
\frac{\mathrm{I}}{\mathrm{I}_{0}}=\frac{1}{\mathrm{~N}+1} \oint_{\mathrm{L}}\left(\beta^{\mathrm{N}} \mathrm{e}^{-\mu \mathrm{Lc}}\right) d L
$$

Where, $\beta(0 \leq \beta \leq 1)$ is the reflection coefficient of gas cell's inner wall and it is only related with reflecting capacity of the inner wall. We can obtain its value through experiments. $\mathrm{N}$ is the number of useful light and it is usually a positive integer which is not greater than ten (When $\mathrm{N}$ is much greater, it indicates that the light we use is reflected again and again, then $\beta^{\mathrm{N}}$ is smaller and the light has little effect on the total light intensity, so this kind of light should be neglected.), L is the length of different light path and other letters in the formula have the same meaning as formula (1).

Pyroelectric detector is the core component of the methane detector and it affects the methane detector's detection precision. Pyroelectric detector we choose is E2V's IR600 and it has better stability and accuracy. IR source is the main part of methane detector. We use lowcost infrared LED in the experiment. In the design, IR source, gas cell's center axis and pyroelectric detector are placed on the same optical axis. With the increasing of IR source and pyroelectric detector service time, their efficiency will drop too, as a result, the signal will decay and the signal of methane concentration will lack fidelity. This design uses the single-light beam and double wavelength differential detection technology to effectively reduce the adverse effects of the above. At the same time, methane detector need not adjust frequently by using the way of referenced measurement.

\section{B. Infrared Methane Concentration Detector Circuit Design}

Circuit is composed mainly by MCU, IR source's modulation circuit, pyroelectric detector, the amplifying and filtering circuit and $\mathrm{A} / \mathrm{D}$ converter, etc. The structure of circuit can be shown in Figure 3.

MCU is the core part of the circuit system and controls the whole system's work, which includes controlling infrared source's modulation, ensuring the start point of data acquisition, processing data of $\mathrm{A} / \mathrm{D}$ converter and computing and displaying the concentration of methane, etc.

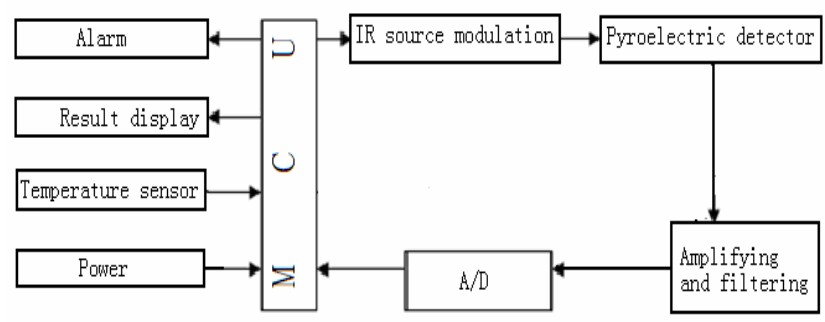

Figure 3. The structure of circuit system

In the system, we use MCU rather than the complex mechanical modulator to control the IR source modulated frequency, and the modulated frequency is $4 \mathrm{~Hz}$. The modulated IR light acts on pyroelectric detector and pyroelectric detector will output weak voltage signal, then the signal will be outputted to the amplifying and filter circuit. The amplifying and filter circuit uses two-stage amplifier circuit. The first amplifier circuit is used to amplify the voltage signal outputted by pyroelectric detector to a suitable amplitude, then the circuit noise will be removed through the active filter circuit, at last the voltage signal is sent to the second amplifier circuit and the signal becomes bigger, then the amplified signal is sent to MCU after becoming digital signal through A/D converter

The temperature detector chip DS1721 is used to gain temperature data and the data is sent to MCU.

The alarm part adopts high decibels buzzer and high brightness LED.

\section{SYSTEM SOFTWARE DESIGN}

The function of software is performing system initialization, gaining temperature data, processing and 
displaying data, etc. The software flow can be shown in Figure 4.

The main program includes data acquisition module, data processing module, signal outputting module and sound-light alarm driver module, etc. After opening the methane detector, the system will initialize the correlated variable and registers related with MCU.

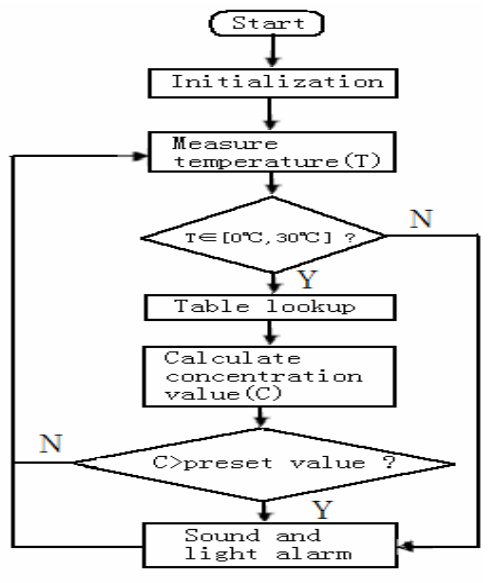

Figure 4. Program flow chart

Temperature value that gained through the chip of temperature detector will be sent to MCU. Methane dectector will send out the alarm signal if the temperature value exceeds the preset value.

In order to display the methane concentration in real time and reduce SCM's workload, the concentration back-induced formul gained through experiments is stored in ROM. Based on gained temperature data, MCU can query related concentration back-in-duced formul, and then it can solve the concentration value through bringing voltage value into the concentration back-in-duced formul. The alarm will send alarm signal, if the concentration value is greater than the preset value.

\section{EXPERIMENT EQUIPMENT, PROCEDURES AND RESULTS}

The experiment equipment is made up of a test chamber, a barometer, a pressure regulator, a thermometer, a temperature controller and a methane detector, etc. Figure 5 shows the whole experiment equipment.

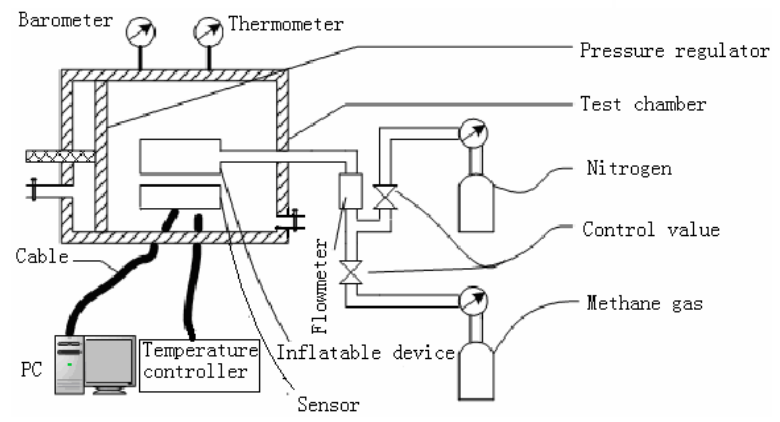

Figure 5. Experiment equipment
Steps as follows:

(1)Clear away the gases in the test chamber, and then pump a certain amount of nitrogen into the chamber.

(2)Keep the chamber's interior temperature at $0.5^{\circ} \mathrm{C}$ through regulating the temperature controller. At the same time, maintain the pressure at $101.325 \mathrm{kPa}$ by adjusting the pressure regulator.

(3)Pump a certain amount of methane gas into the chamber, then shut the control valve and keep the pressure at $101.325 \mathrm{kPa}$; at last we should measure and record the methane concentration.

(4)Change the methane concentration, and then repeat the step (3).

(5)Increase $1^{\circ} \mathrm{C}$ at step (2). If the temperature value is less than $30^{\circ} \mathrm{C}$, repeat the step (1)-(4).

In order to satisfy the requirement of coal mine safety regulation, the range of temperature is settled in $0{ }^{\circ} \mathrm{C}-30^{\circ} \mathrm{C}$ in the experiment.

Based on the explanatory notes in the instruction book, the reflection coefficient $\beta$ of gas cell inner wall is 0.8 , the effective length of gas cell is $7.0 \mathrm{~cm}$ and the inner diameter of is $1.6 \mathrm{~cm}$. In the range of $\left(18^{\circ} \mathrm{C}-20^{\circ} \mathrm{C}\right]$, we can gain the data as table I. The way of obtaining data is calculating the average value of data at $18.5^{\circ} \mathrm{C}$ and $19.5^{\circ} \mathrm{C}$, and the way of obtaining data is similar at other temperature range.

TABLE I . THE CORRESPONDING RELATION TABLE OF METHANE CONCENTRATION(\%) AND VOLTAGE VALUE(V)

\begin{tabular}{|c|c|c|}
\hline Actual concentration & Voltage measured & Concentration measured \\
\hline 0 & 4.82 & 0.012 \\
\hline 0.5 & 4.59 & 0.498 \\
\hline 1.0 & 4.41 & 0.988 \\
\hline 1.5 & 4.25 & 1.504 \\
\hline 2.0 & 4.11 & 2.020 \\
\hline 2.5 & 4.01 & 2.488 \\
\hline 3.0 & 3.86 & 3.103 \\
\hline 3.5 & 3.78 & 3.511 \\
\hline 4.0 & 3.69 & 3.995 \\
\hline 4.5 & 3.59 & 4.514 \\
\hline 5.0 & 3.50 & 5.030 \\
\hline & & \\
\hline
\end{tabular}

Based on data in table I, we can gain the fitting formulas related to voltage (V) and methane concentration (C): $\mathrm{C}=1.56 \mathrm{~V}^{2}-16.78 \mathrm{~V}+44.6$.

When the temperature is less than $18^{\circ} \mathrm{C}$, we use every $3{ }^{\circ} \mathrm{C}$ as a temperature range and the error is $0.27 \%$. When the temperature is less $30^{\circ} \mathrm{C}$ and greater $18^{\circ} \mathrm{C}$, we use every $2^{\circ} \mathrm{C}$ as a temperature range and the error is $0.23 \%$. In the range of $0{ }^{\circ} \mathrm{C}$ and $30^{\circ} \mathrm{C}$, the fitting formulas related to voltage and methane concentration is shown in table II. 
TABLE II . THE FITTING FORMULAS RELATED TO VOLTAGE AND CONCENTRATION IN DIFFERENT TEMPERATURE RANGE

\begin{tabular}{|l|l|}
\hline Temperature range & The fitted formulas \\
\hline$(0,3]$ & $\mathrm{C}=1.02 \mathrm{~V}^{2}-12.38 \mathrm{~V}+36.25$ \\
\hline$(3,6]$ & $\mathrm{C}=1.09 \mathrm{~V}^{2}-12.99 \mathrm{~V}+37.54$ \\
\hline$(6,9]$ & $\mathrm{C}=1.16 \mathrm{~V}^{2}-13.60 \mathrm{~V}+38.74$ \\
\hline$(9,12]$ & $\mathrm{C}=1.26 \mathrm{~V}^{2}-14.40 \mathrm{~V}+40.29$ \\
\hline$(12,15]$ & $\mathrm{C}=1.36 \mathrm{~V}^{2}-15.19 \mathrm{~V}+41.71$ \\
\hline$(15,18]$ & $\mathrm{C}=1.38 \mathrm{~V}^{2}-15.27 \mathrm{~V}+41.67$ \\
\hline$(18,20]$ & $\mathrm{C}=1.56 \mathrm{~V}^{2}-16.78 \mathrm{~V}+44.67$ \\
\hline$(20,22]$ & $\mathrm{C}=1.56 \mathrm{~V}^{2}-16.75 \mathrm{~V}+44.38$ \\
\hline$(22,24]$ & $\mathrm{C}=1.58 \mathrm{~V}^{2}-16.74 \mathrm{~V}+43.93$ \\
\hline$(24,26]$ & $\mathrm{C}=1.58 \mathrm{~V}^{2}-16.65 \mathrm{~V}+43.43$ \\
\hline$(26,28]$ & $\mathrm{C}=1.59 \mathrm{~V}^{2}-16.67 \mathrm{~V}+43.12$ \\
\hline$(28,30]$ & $\mathrm{C}=1.59 \mathrm{~V}^{2}-16.56 \mathrm{~V}+42.62$ \\
\hline
\end{tabular}

\section{CONCLUSION}

Based on other people's research, we design an infrared methane concentration detector which not only uses the direct light but also considers the effect of reflected light to pyroelectric detector. In order to reflect the relationship, between changes in temperature and methane concentration, the design uses a single strike at different temperature ranges of methane concentration and voltage to conduct fitting formula. It is with higher detection accuracy than using temperature compensation circuit in literature [11] and [12]. The result of experiment demonstrates that the methane concentration detector designed in this paper can satisfy the requirement of coal mine safety production.

\section{ACKNOWLEDGEMENT}

This work is supported by the National Natural Science Foundation of China (No.51274078) and the Fundamental Research Funds for the Central Universities.

\section{REFERENCES}

[1] Haibo Liu, Jing Shen, "Research Advances on Catalyzing Burning Gas Sensors," Intelligent Computer and Applications, 2011, 1(3), pp.71-73.

[2] Xuemei Fan, "On the applicability of methane gas sensor detecting by using the thermal catalytic combustion," Science and Technology of Datong Coal Mining Administration, 2009, No.121, pp.19-22

[3] Haidong Ding, Yulong Zhao, Zhi Sun, "Research Development of Oxide Semiconductor Methane Sensor," Coal Science and Technology, 2005, 33(7), pp.69-71

[4] Suling Wang, "The use of optical interference sensor and carrier of catalyst sensor alarm in real life," ShanXi Coal, 2010,30(12), pp.6264

[5] Xinqi Song,Chunxia Yu,Haiyang Li, "Design of a New Laser Gas Sensor[J] Computer Measurement \& Control," 2011,19(8), pp.19561957

[6] D Richter A, Fried, Wert B p, etal, "Development of a tunable midIR difference frequency laser source for highly sensitive airborne tracegas detection," Appl.Phys.B.2002, 75,pp.281-288.

[7] Okaiima H, Kakuma S, Uchida K, eta1, "SICE-ICA International Jiont Conference 2006, "Busan, Korea, October 18-21, 2006.

[8] Mengran Zhou,Zhenbi Li, etal, "Studies on Coal Mine Gas Optical Fiber Sensor Based on Spectrum Absorption," Safety in Coal Mines, 2007 (8), pp.6-9.

[9] Brown L R, Chris D Benner, Champion J P, etal, "Journal of Quantitative Spectroscopy and Radiative Transfer," 2003(82), pp.219.

[10] Rongxuan Li, "Design of Testing System Based on a New NonDispersive Infrared Gas Quantitative Detection Model," Hefei University of Technology, 2011

[11] Dongxu Liu,Zhibing Wang,Jilong Zhang,etal, "Design of Faint Signal Processing Circuit Based on Infrared Methane Sensor," Instrument Technique and Sensor, 2010(6), pp.69-71

[12] Zhengjie Zhao,Dongxu Liu,Jilong Zhang,etal, "The System Design of NDIR Gas Sensors Based on Electrical Modulation," Spectroscopy and Spectral Analysis, 2011, 31(2), pp.570-572 